\title{
ASPECTOS PSICO-SOCIOLÓGICOS DEL USO DE INTERNET
}

\section{Tomàs Baiget}

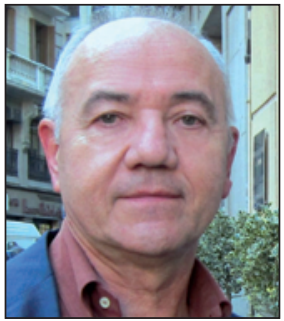

Tomàs Baiget ha puesto en marcha y/o trabaja en varios proyectos tales como la revista $E$ I profesional de la información (EPI), las listas electrónicas IweTel e Incyt, el think tank Grupo de Análisis sobre Estrategia y Prospectiva de la Información (ThinkEPI), el Directorio de Expertos en el Tratamiento de la Información (EXIT), el Centro Internacional para la Investigación en Estrategia y Prospectiva de la Información (Ciepi), el sistema International Registry of Authors-Links to Identify Scientists (IraLIS) y el blog Quotes \& Jokes para aprender inglés. Es miembro del Comité Asesor de la Online Information Conference, Londres, y organizador del Spanish Meeting Point; editor del repositorio E-prints in Library and Information Science (E-LIS); y profesor del Master Online de Documentación Digital, de la Universitat Pompeu Fabra. Sus líneas de investigación son: comunicación científica, networking, acceso abierto, futuro profesional y mercado de la información.

El profesional de la información EPI SCP, Apartado 32280, 08080 Barcelona baiget@sarenet.es http://www.baiget.com

\section{Resumen}

El crecimiento de la población, la desaparición de fronteras, la facilidad de transporte y sobre todo las redes de telecomunicaciones han dado lugar a que las personas interactuemos mucho más y se acentúe nuestro carácter de sociedad humana dentro de un contexto general de globalización. Por otra parte, el uso de los medios de comunicación y de las redes sociales ha propiciado un mayor intercambio de información, y una mayor cooperación, incluso entre personas desconocidas. Estos fenómenos tienen implicaciones psicológicas importantes para los individuos en general, y en especial para los bibliotecarios-documentalistas, unos profesionales que deben tener importantes dotes y habilidades para tratar con sus usuarios.

\section{Palabras clave}

Sociedad, Humanidad, Comunidades, Redes, Internet, Uso, Comunicación, Networking, Web 2.0, Psicología, Sociología, Comportamiento, Capital social.

\section{Title: Psycho-sociological aspects of internet use}

\begin{abstract}
Population growth, disappearance of borders, ease of transport services, and particularly telecommunications networks have led people to interact much more with each other and to accentuate the social aspect of humankind within the general context of globalization. Moreover, the use of media and social networks has led to greater information exchange and closer cooperation, even among strangers. This phenomenon has important psychological implications for individuals in general and especially for librarians and information scientists, professionals who must have good skills to deal with users.
\end{abstract}

\section{Keywords}

Society, Humankind, Communities, Networks, Internet, Use, Communication, Networking, Web 2.0, Psychology, Sociology, Behaviour, Social capital.

Baiget, Tomàs. "Aspectos psico-sociológicos del uso de internet". El profesional de la información, 2011, enero-febrero, v. 20, n. 1, pp. 87-93.

\section{Hacia una sociedad global}

Las comunidades y las sociedades crean órganos de gobierno, en quienes se delega el papel de dirección y gestión de los intereses generales. Las naciones crean el Estado.

Artículo recibido el 13-11-10

Aceptación definitiva: 02-12-10
Entre el Estado y la sociedad se encuentra la llamada esfera pública, que es "una red para comunicar información y puntos de vista" (Habermas, 1996). La esfera pública es una componente esencial de la organización socio-política porque es el espacio donde la gente se reúne como ciudada- 
nos y expresan sus opiniones para influir en las instituciones políticas de la sociedad.

En nuestra sociedad-red la esfera pública se organiza sobre la base de las redes de comunicación, tanto internet como los medios de difusión (Castells, 2008).

La sociedad civil es la expresión organizada de estos puntos de vista, siendo la relación entre el Estado y la sociedad civil la piedra angular de la democracia. Sin una efectiva sociedad civil capaz de estructurar y canalizar debates ciudadanos sobre diversas ideas e intereses en conflicto, el Estado se aleja de sus súbditos.

Por otra parte, una serie de cuestiones que afronta la humanidad son globales en sus manifestaciones y en su tratamiento: gestión del medio ambiente con el daño causado por el desarrollo (por ejemplo, el calentamiento global) y la necesidad de contrarrestar este deterioro con una estrategia global, la conservación de la información a largo plazo, la globalización de los derechos humanos y la emergencia de la cuestión de la justicia social para el planeta en general, y la seguridad mundial como un problema común, incluida la proliferación de armas de destrucción masiva, el terrorismo global y la práctica de la política del miedo bajo el pretexto de luchar contra el terrorismo...

La disminución de la capacidad de los sistemas políticos a escala nacional para manejar los problemas del mundo a escala global ha provocado el surgimiento de una sociedad civil global (Castells, 2008).

\section{Las personas como nodos sociales}

\section{Concepto de presencia}

La presencia se define como el sentido de "estar ahí". Existen diferentes conceptos de presencia que se pueden agrupar en dos categorías: física y social. La primera se refiere a la sensación de estar físicamente situado en algún lugar e implica que el medio parece ser invisible; la social se refiere a ser y comunicarse con alguien, con la implicación de que el medio parece estar transformado en una entidad social. Aunque con frecuencia se distingue entre la presencia física y la social, en realidad se trata de sinónimos, o al menos son estrechamente interdependientes.

La división es útil para decidir mejor sobre la forma adecuada de la presencia que debe tenerse en cuenta en el diseño de un sistema y dar lugar a los resultados deseados por el uso de una tecnología. Además, la división entre física y social permite una reconceptualización de la presencia social, cosa que tiene importantes consecuencias para la representación de grupos en ambientes virtuales y en internet (Rogers; Lea, 2005).

El factor crítico en un medio de comunicación es la "presencia social", definida como una cualidad subjetiva del medio. La presencia social se concibe como el grado de contacto interpersonal que permite un medio y está estrechamente relacionada con cuestiones como la "intimidad" y la "inmediatez". Clasificados según su grado de "presencia social" diferentes medios de comunicación se observó que el cara a cara tiene el valor mayor, luego le siguen otros medios como televisión, teléfono, etc., y, por último, las cartas co- merciales. El correo electrónico y los documentos con sólo texto ocupan un lugar relativamente bajo en el ranking (Spears; Lea, 1992), mientras que los entornos de realidad virtual ocuparían un puesto relativamente alto (Rogers; Lea, 2005).

En aplicación de estos conceptos, la revista El profesional de la información (EPI) aumenta la presencia de los autores de sus artículos en la comunidad de bibliotecarios-documentalistas publicando sus retratos. También ha implementado el Directorio EXIT (Expertos en el tratamiento de la información) como herramienta de vertebración de la citada comunidad profesional (Orduña-Malea et al., 2007). En la web de EPI todos los autores de los artículos publicados están enlazados a sus fichas de EXIT, obteniéndose así una malla de autores dentro del colectivo de bibliotecarios, documentalistas y comunicadores.

Con nuestra presencia, aunque sea por simple estigmergia -imitación inconsciente- (Peset et al., 2008), influimos en quienes nos rodean. Existen medios de comunicación muy poderosos para causar impacto sobre las otras personas como es la TV pero sólo está al alcance de muy pocos. Y además quien más influye sigue influyendo aún más (efecto Mateo). Con la TV existía una brecha entre los televisibles y todos los demás, pero internet ha venido a dar una oportunidad a casi toda la población sin apenas rupturas ni solución de continuidad, al menos entre la clase media.

Debemos tener presencia en la Red, definir nuestra identidad y ocupar nuestro lugar en ella

\section{Identidad}

Se llama identidad la cualidad o condición de ser uno mismo en sustancia, composición, naturaleza, propiedades o cualidades. La unicidad de ser de una persona o cosa en todo momento y en cualquier circunstancia, la condición o hecho de lo que una persona o cosa es en sí misma y no otra.

¿Qué impacto tienen las tecnologías de la información sobre nuestra identidad, individualidad y personalidad? Ha sido señalado por muchos autores que la ampliación o el incremento de las redes sociales ha creado un nuevo sentido de identidad para determinadas personas, por ejemplo, los adolescentes (Lorente, 2002). En particular, el hecho de que el teléfono móvil sea algo personal ha hecho que su uso se haya convertido en un factor importante en la individualidad de sus usuarios.

El sentido de pertenencia, tan fundamental para nosotros, tiene cuatro componentes clásicos: lugar, familia, país y raza. Las tecnologías móviles han tenido un impacto sin duda en los dos primeros. El sentido de pertenencia a un lugar va dando paso lentamente al sentimiento de pertenencia a la red de comunicaciones. "Los elementos emocionales relacionados con el espacio se pierden y se transfieren a un nivel social, que es la lealtad, el sentido de identificación, la familiaridad, la estabilidad, la seguridad, y otros" (Fortunati, 2000). Los teléfonos móviles per- 
miten a los usuarios construir su propio ambiente 'de mi casa', independientemente del lugar en que se encuentran físicamente. Con el teléfono de línea fija, una llamada entrante se dirige a un grupo o familia. Con el móvil, es una persona la que está siendo llamada, independientemente de su lugar (Srivastava, 2005). Los conceptos de país y raza también se ven influenciados poco a poco por la desaparición de fronteras y las migraciones.

Dentro del movimiento de interconexión con los demás, cada persona es un nodo de la red, lo que refuerza la idea de formar parte de la misma, de compararse con los demás nodos, de recibir y transmitir información, y de mantener ese puesto de una forma responsable. No podemos prescindir de nuestra condición de seres sociales. Incluso podríamos esgrimir una obligación ética de no ser pasivos y colaborar para que la sociedad funcione. Todos tenemos conocimientos que aportar a nuestros semejantes, no deberíamos ser agujeros negros absorbiendo información y experiencias y no ofreciendo nada a los demás.

Ser nodos de la red conlleva el mantenimiento de una identidad clara y de unas acciones de presencia para que se nos conozca. Tales acciones dependerán de nuestra extroversión, intereses, proactividad..., y pueden ser de imagen, de marketing personal y profesional, filantrópicas, etc. Aguillo (2010) recomienda que todos los profesionales que tienen algo que aportar a la sociedad (investigadores, documentalistas, etc.) se construyan una web, bien diseñada, donde muestren sus conocimientos y ofrezcan sus trabajos.

Algunas personas padecen problemas de timidez, o sienten ciber-agorafobia, o se sienten inseguras en internet, o desconfían excesivamente y súper-protegen su intimidad, pero dadas las cifras de utilización de la Red son una clara minoría. Evidentemente, participar en la sociedad tiene riesgos, y la máxima seguridad personal sólo se obtendría viviendo en un búnker.

Todos tenemos algo que aportar, no deberíamos ser agujeros negros absorbiendo información y experiencias y no ofreciendo nada

\section{La firma}

Un aspecto que puede parecer secundario, y en cambio es de especial relevancia en la comunicación, es la forma de firmar. Hay que dar mucha importancia a la firma, que es nuestra marca o descriptor personal. Firmar siempre igual y en el formato internacional nos facilitará la búsqueda de nuestra bibliografía, y también que sea fácil que nos localicen para participar en proyectos, cursos, conferencias, oportunidades de trabajo (Baiget et al., 2007).

El hecho de mentalizarnos y asumir que somos ciudadanos del mundo, nos lleva ineludiblemente a ser más serios. Ya no podemos seguir parapetados tras unas murallas, haciendo nuestras capillitas, al amparo de un idioma local. Publicar en la web los resultados en inglés de nuestra investigación nos sitúa junto a cualquier investigador de Massachusetts o de
Japón. Ya no vale esa idea de "ellos, los americanos, allá lejos, desconocidos, y nosotros aquí con nuestras cosas", con un escondido complejo de inferioridad, y como si fuéramos de mundos diferentes.

Muchas tesis españolas se han hecho como un trámite burocrático, sin pretender ser los primeros del mundo en investigar algo

\section{Tomar conciencia de ser ciudadanos del mundo}

De las muchas cosas importantes que están cambiando, una de ellas es la de convertirnos poco a poco en ciudadanos del mundo.

La ciencia es un producto universal, pero al depender del idioma en que se escribe se encuentra a veces con más dificultades para traspasar las fronteras que las mismas personas. Para los españoles, que tanto hemos defendido el castellano y demás lenguas vernáculas, nos resulta bastante frustrante que para lograr que nuestras aportaciones científicas formen parte de la cresta de la ola mundial las tengamos que publicar en inglés. Todo lo que actualmente no se publique en inglés sólo puede optar a ser "divulgación local", a no incrementar el estado del arte de la ciencia, a pasar sin pena ni gloria.

España ha vivido muchos años de espaldas a la Ciencia, y -salvo notables excepciones- muchos científicos españoles han ejercido con cortedad de miras. Muchas tesis se han hecho como un trámite burocrático, sin pretender ser los primeros del mundo en investigar algo. Ha sido una postura derrotista -sin plantearnos competir con norteamericanos, ingleses o alemanes-, tal vez por falta de medios, por desconocimiento de idiomas, o por incapacidad de conocer lo que ya está inventado (lo que incluye también a los propios directores de tesis y a los tribunales), o de salir del paso con una tesis del tipo "contar patas de hormiga".

En biblioteconomía-documentación poca gente se ha tomado la ciencia en serio, investigando cosas útiles y procurando después que los resultados se difundieran de verdad. A veces parece como si la gente hiciera ciencia como una religión, como una oración introvertida, dejando un trabajo hecho quizá "para la posterioridad". Sin marketing de los resultados, ese trabajo de meses o de años sólo habrá sido un proceso burocrático para obtener un título, y una miserable pérdida de tiempo y de esfuerzo que no hace crecer la Ciencia de verdad. No se ha tenido el objetivo de escribir algo que sirva e impacte al resto de la Ciencia universal, y muchísimo menos inventar algo patentable y que enriquezca a nuestro país.

Sin embargo, con la actual situación de globalización muchos ya empiezan a tener vergüenza de escribir los hasta hace poco frecuentes artículos de refritting ${ }^{2}$ para publicar en las revistas españolas. Por un lado, los refritos son bastante fáciles de desenmascarar gracias a Google y a los múltiples sistemas de detección de plagio y auto-plagio que existen. Por otro, muchas revistas españolas publican en in- 
glés. La revista EPI publica en español, pero en cada número acostumbra a incluir un artículo en inglés como aviso a navegantes: "no hace falta que nos cueles traducciones del inglés". Y por último los investigadores se van conociendo, tanto a través de las redes como personalmente, ya no son anónimos, y el pirateo de los trabajos de un colega hasta puede resultar violento.

\section{Conceptos y aspectos sociales}

Las redes sociales existen porque los seres humanos son sociales y requieren relaciones con otros seres humanos para sobrevivir. Las relaciones son fundamentales para el bienestar psicológico, lo que ha sido bien documentado a lo largo de los años y no sólo sigue siendo interesante para los investigadores de hoy en día (Durden et al., 2007; Henderson, 1977), sino que además su interés va en aumento.

En los años recientes todos hemos descubierto el extraordinario impacto y auge de las redes sociales online, como Facebook. La mayoría de esos sitios fomentan la comunicación con los demás, proporcionando directorios de poblaciones de usuarios pertinentes, oportunidades de auto-descripción, cargar (upload) contenidos, sistemas de recomendación, etc. Algunos investigadores sugieren que fomenta la "presencia conectada", que los usuarios de internet tienen redes sociales mayores que los no usuarios, y que internet ayuda a mantener la red social de cada uno (Ellison et al., 2007).

Las webs de redes sociales están prosperando gracias a que permiten mostrar las habilidades de las personas y las invitan a ser creativas y expresivas. Se pueden ver y compartir fotos y presentaciones en vídeo. Hacen falta pocos conocimientos para publicar aspectos seleccionados de la propia vida.

De todas formas el encuentro social humano es algo multisensorial, y la comunicación basada en la tecnología no es capaz de simularlo completamente. Las conversaciones cara a cara y el tiempo invertido en la compañía física seguirán determinando el crecimiento de las redes sociales sólidas y reales tanto familiares como de amistad (Coyle; Vaughn, 2008). Sin embargo los sitios de redes sociales pueden ayudar a generar nuevas amistades y colaboraciones, pues permiten seleccionar rasgos y características de compatibilidad de un gran número de personas, cosa que sería imposible hacer en persona.

Al principio se difundieron visiones pesimistas de las redes, pues se temía que producirían una alienación de la gente al apartarse de los medios convencionales de comunicación, como el cara a cara. Quizá influyó en ello la imagen de los geeks usuarios iniciales, personas inmersas en las tecnologías que parecían estar lejos del mundo real. Contrariamente, también se manifestaron opiniones utópicas considerando internet como un ágora electrónica independiente capaz de sustituir cualquier tipo de encuentro físico o de lugar de trabajo. El debate se basaba en una dicotomía simplista entre los modos de comunicación online y real, que se consideraban como cosas distintas y sin relación.

Sin embargo estudios más recientes demuestran que la comunicación mediada por ordenador y la comunicación móvil son sólo dos formas de comunicación entre otros muchas, y que las relaciones sociales que se originan en la interacción online continúan y se alternan en el mundo real y viceversa. Además las redes, ya sean de parentesco, amistad, trabajo o de simple contacto, se entrelazan sin solución de continuidad.

Las relaciones sociales que se originan en la interacción online, continúan y se alternan en el mundo real, y viceversa

Las posibles interferencias trabajo-vida privada también se vieron como algo peligroso y abominable, pero hoy muchos de nosotros nos hemos habituado a ello, por ejemplo en Facebook, incluso con placer, en especial si nuestro trabajo nos satisface.

Los móviles también generaron una gran desconfianza cuando aparecieron. Además de ser considerados gadgets para pijos, nadie quería dar sus números para que no le molestaran durante sus sacrosantas horas de descanso, pero esto se ha demostrado ser también falso. La gente tiene sentido común y nadie llama a un colega en horas intempestivas para algo irrelevante.

Los círculos sociales que los individuos generan y mantienen con la ayuda de la información y la comunicación introducen nuevas cualidades en el concepto de "comunidad". Por tanto, es imprescindible tener un enfoque integral y holístico que reconozca la diversidad y el carácter híbrido de la "ecología comunicativa" (Foth; Hearn, 2007).

Apenas hemos empezado a percibir el impacto innovador de las redes sociales, del conglomerado de sus interacciones y aprendizajes recíprocos. El entramado social será distinto (Punset, 2010).

\section{Capital social}

Es la capacidad de un grupo humano para que prospere la colaboración y se generen oportunidades de realizar trabajos conjuntos que produzcan beneficios tanto individuales como colectivos. Se basa en tres elementos principales: la confianza mutua, las normas consensuadas eficaces y las redes sociales.

Los individuos también tienen un capital social según su habilidad de establecer relaciones con los demás y recibir beneficios de ellas. Una persona con más capital social es probable que se desarrolle mejor psíquica, social, emocional, académica y físicamente.

Según el tipo de relaciones se distingue entre capital social de consolidación hacia nuestra propia comunidad (bonding social capital) y de prospectiva de nuevas relaciones, con enlaces hacia el exterior (bridging social capital). Algunos consideran también la habilidad de mantener conexiones estables con comunidades nuevas a las que antes no se pertenecía como un tipo intermedio de capital social (maintaining social capital). 


\section{Conceptos y aspectos psicológicos}

En la sección anterior hemos visto una serie de facetas y fenómenos que tienen lugar en las redes sociales. Ahora veremos sus efectos sobre los individuos.

Como se dijo, el capital social se refiere en general a los beneficios que recibimos de nuestras relaciones sociales. Está relacionado con resultados tan diversos como la promoción profesional, el éxito dentro de una organización, una mejor salud pública, reducción de los índices de criminalidad... A nivel psicológico y de bienestar físico diferentes estudios demuestran que las personas con más capital social son más propensas a tener comportamientos que conducen a disfrutar de mejor salud, desarrollo emocional y éxito académico.

Las personas con más capital social tienden a tener mejor salud, desarrollo emocional y éxito académico

Para acumular capital social los individuos necesitan mantener sus relaciones, hacer inversiones de tiempo y dedicación, de lo cual, consciente o inconscientemente, esperan un determinado tipo de retornos a corto y largo plazo. Los sitios de redes sociales como Facebook facilitan la creación y el mantenimiento de lazos, cosa que justifica su éxito. Según Ellison et al. (2007) el uso de Facebook resultó muy positivo para distintas medidas de capital social, tanto con enlaces externos débiles (bridging social capital) como internos con los amigos y la familia (bonding social capital). Por otra parte, los jóvenes con baja autoestima se benefician más del uso de Facebook que aquellos con una mayor autoestima, pues este sitio web facilita la comunicación, especialmente en las interacciones sociales iniciales para acercarse a la gente, y mitiga el miedo al rechazo.

Similarmente a lo dicho antes cuando se trataron los aspectos sociales de las redes online, los primeros estudios del impacto de internet sobre la sociabilidad decían que su uso restaba tiempo de contacto con la familia y los amigos, y que como consecuencia los usuarios eran más propensos a la depresión. Se argumentaba que esto se debía a que los vínculos (débiles) generados online estaban reemplazando los lazos (fuertes) de la familia y los amigos.

Probablemente los primeros estudios se hicieron sobre comunidades creadas mediante el simple intercambio de textos, que permiten más el anonimato. Las redes multimedia como Facebook son mucho más transparentes, pues aunque los usuarios siempre se pueden poner 'caretas' o falsear ciertos datos, en general se aporta mucha información personal a través de fotos, vídeos, gustos, pensamientos, afiliaciones, red de contactos, actividad laboral, etc. Quizá sea necesario recordar al lector que Facebook permite diferentes niveles de privacidad y restricción de acceso a los diferentes objetos informativos que pongamos en nuestras páginas.

En un primer estudio, Kraut et al. (1998) percibieron efectos nocivos en el uso de las redes pero posteriormente este mismo autor (Kraut et al., 2002) encontró que cuando se examina un período más largo de tiempo, el uso de internet ya no estaba asociado con disminución de la comunicación y de la participación en la familia (y de las medidas asociadas a la soledad y la depresión). Los efectos del uso de las redes sociales online fueron en general positivos, tanto para los introvertidos como -sobre todo- para los extravertidos. Otros investigadores también sostienen que el uso de internet tiene efectos positivos sobre el bienestar psicológico.

Posiblemente entre los primeros internautas había una proporción mucho mayor de geeks, con su clásica imagen tópica de piel blanca por no despegarse nunca de su ordenador. Estudios posteriores lo han desmentido, siendo cada vez más evidente que los usuarios de internet son tan sociables como los no usuarios, si no más, y que internet se utiliza a menudo como una herramienta para mantener y ampliar las relaciones sociales (Boase et al., 2006; Rice et al., 2007).

\section{Apoyo social}

Se define como "el intercambio de mensajes verbales y no verbales que transmiten emociones, información o referencia, para ayudar a reducir la incertidumbre y el estrés" (Walther; Boyd, 2002).

El apoyo social se obtiene sobre todo a través de los enlaces fuertes con los familiares y amigos íntimos, pero se ha estudiado el apoyo (o no) recibido por los enlaces débiles de las redes sociales. Entender los factores y procesos que conducen a la prestación de apoyo social es un área importante de la investigación sociológica y psicológica, pues promete obtener técnicas para la prevención y el combate de problemas como la ansiedad, el estrés y la depresión.

El apoyo social se puede recibir en forma de consejos y recomendaciones desde las redes sociales y listas de correo: por ejemplo novedades tecnológicas interesantes, webs con información, recetas, etc., lugares para mejorar el trabajo o lugares de ocio.

Las webs de redes sociales y las listas de correo permiten recibir apoyo social en forma de consejos y recomendaciones

Valkenburg et al. (2006) encontraron que cuanto más se utilizan los sitios de redes sociales, mayor es la frecuencia de interacción con los amigos, lo cual aumenta la autoestima y, en última instancia, la satisfacción con la vida. Curiosamente, el hecho de ayudar a los demás, dando soporte técnico incluso a desconocidos, mejora también el autoestima (Constant et al., 1996).

Igualmente los internautas esperan tener apoyo social sobre los productos y servicios que compran (Lin et al., 2009).

Es importante que las bibliotecas tengan en cuenta este comportamiento social que cada vez más se produce por la Red para proveer también apoyo.

Cuanta más experiencia y veteranía en internet, las personas crean más relaciones online. Es decir, que con una mayor familiaridad con internet se reduce la percepción de riesgo y se tiene mayor confianza para participar en las redes sociales. El tiempo dedicado a los espacios de redes sociales 
online facilita el desarrollo de relaciones sociales con otros usuarios (Di Gennaro et al., 2007).

Las redes sociales online, no sólo son mucho mayores que las redes presenciales, sino que tienen una estructura distinta, pues no se ven influenciadas por factores demográficos como nivel económico, o el atractivo físico, etc.

La red de la vida social real está formado por miembros de la familia, parientes, vecinos y colegas, en cambio un usuario medio de Facebook además tiene enlaces de tipo débil o externos. Un estudio realizado en 2002 halló que la media de contactos en Facebook era de 217, una vez y media el número esperado en la vida real (Hill; Dunbar, 2003). Otros estudios demuestran que el número de contactos aumenta constantemente. Ello es debido a la ya citada percepción de menor riesgo de los internautas con el uso continuado de la red con lo que es más fácil la aceptación de nuevos miembros, la solicitud de membresía, existe menor propensión al rechazo, mayor sentimiento de ser popular, y no se eliminan contactos con los cuales ya no se tiene relación.

Las mujeres y los hombres actúan de forma diferente en las redes sociales. El número de enlaces de las mujeres es mayor, pasan más tiempo comunicándose y les preocupa menos agregar nuevas personas a su red, aunque no sepan mucho de ellas. Aunque las mujeres se espera que sean más estrictas y selectivas en ambientes de comunicación online, los resultados demuestran que reciben más solicitudes de amistad y pokings (toques en el hombro, en terminología de Facebook) y los aceptan en mayor proporción que los hombres.

Cuanto mayor es la autoestima, menor es el porcentaje de desconocidos o poco conocidos por el usuario. Las personas con alta autoestima son reacias a añadir nuevos contactos en su red a menos que los conozcan. Parece que las personas con poca autoestima sienten más necesidad de comunicarse con desconocidos (Acar, 2008).

Estos resultados nos ayudan a comprender mejor por qué la gente participa en la creación de redes sociales online y cómo le afecta esta actividad.

Las personas con poca autoestima sienten más necesidad de comunicarse con desconocidos

\section{Conclusiones}

Los bibliotecarios-documentalistas se ven obligados a evolucionar para adaptarse a la nueva situación marcada por las TICs, deben ser más comunicadores que antes, más proactivos, y deben hacer mejor marketing para demostrar su valía y no ser desplazados del mundo laboral.

También deben actuar a través de las redes, manejando con soltura los nuevos canales de comunicación social como blogs, Twitter y Facebook. Las encuestas demuestran que cada vez hay más gente "viviendo en la Red", comunicándose en el nuevo medio. Ahí hay que estar como profesionales.

Ante esta situación en este trabajo hemos querido asomarnos a los estudios actuales sobre psicología y sociología de grupos y redes, para poder sacar pautas de comportamiento y entender los mecanismos de colaboración, tanto presencial como en la Web. Lo que hemos hallado es bastante general, y no siempre de aplicación directa al trabajo del profesional de la información, pero nos proporciona una marco de conocimiento general muy útil.

Estar trabajando en un mundo global que se hace pequeño nos obliga a interaccionar (¿y cooperar?) con otras muchas personas. Todos somos nodos (identificados por nuestra firma, nuestra IP, nuestra web personal, nuestra página en Facebook) de las redes mundiales, no vivimos aislados.

Con nuestra participación en las redes vamos incrementando nuestro capital social, lo cual (supuestamente) nos servirá para recibir ayuda tanto en lo personal como en lo profesional el día que lo necesitemos. La Red es una reproducción online de la vida real, por lo que hay que pensar que la inmensa mayoría de la gente tiene buenas intenciones. En términos evolucionistas seguramente la sociedad no es perversa, pues si no ya nos habríamos autodestruido. Se observa que las personas adquieren confianza en internet a medida que van participando más.

La sociedad se va moldeando y adaptando a la redes sociales, cuyo impacto no ha hecho más que insinuarse. No hay que descuidarse, pues como en todas partes puede haber delincuentes, pero las redes van a proporcionar un enorme cambio de mentalidad y de comportamiento social, y los bibliotecarios -una profesión con altos componentes social y psicológico- se verán muy influenciados por ellos.

\section{Nota}

1. Palabra usada por Isidro F. Aguillo para referirse a los artículos realizados a base de refritos de textos originales en inglés.

\section{Bibliografía citada}

Acar, Adam. "Antecedents and consequences of online social networking behavior: the case of Facebook". Journal of website promotion, 2008, v. 3, n. 1, pp. 62-83. DOI: $10.1080 / 15533610802052654$

Aguillo, Isidro F. "Reflexiones y buenas prácticas sobre la presencia personal en la Web académica". Anuario ThinkEPI, 2010, n. 4, pp. 269-271.

Baiget, Tomàs; Rodríguez-Gairín, Josep-Manuel; Peset, Fernanda; Subirats, Imma; Ferrer, Antonia. "Normalización de la información: la aportación de IraLIS". El profesional de la información, 2007, nov.-dic., v. 16, n. 6, pp. 636-643.

DOI: 10.3145/epi.2007.nov.10

Boase, Jeffrey A. America online and offline: the relationship of personal networks to email and other communication media. Doctoral thesis, Univ. of Toronto, 2006. http://web.mac.com/jboase/professional/files_files/Ame rica Online and Offline - Doctoral Dissertation - Boase.pdf

Castells, Manuel. "The new public sphere: global civil society, communication networks, and global governance". The annals of the American Academy of Political and Social Science, 2008, n. 616, p. 78.

DOI: $10.1177 / 0002716207311877$ 
Constant, David; Sproull, Lee; Kiesler, Sara. "The kindness of strangers: The usefulness of electronic weak ties for technical advice". Organization science, 1996, n. 7, pp. 119-135.

Coyle, Cheryl L.; Vaughn, Heather. "Social networking: communication revolution or evolution?". Bell Labs technical journal, 2008, v. 13, n. 2, pp. 13-18.

DOI: $10.1002 /$ bltj.20298

http://www.alcatel-lucent.com/enrich/v3i12009/pdf/BLTJ_ Social_Networking_-_Communication_Revolution_or_Evolu tion.pdf

Di Gennaro, Corinna; Dutton, William H. "Reconfiguring friendships. Social relationships and the internet". Information, communication \& society, 2007 , Oct., v. 10 , n. 5 , pp. 591-618.

DOI: 10.1080/13691180701657949

Directorio de Expertos en el Tratamiento de la Información (EXIT) http://www.directorioexit.info

Durden, Emily D.; Hill, Terrence D.; Angel, Ronald J. "Social demands, social supports, and psychological distress among low-income women". J. social and personal relationships, 2007 , v. 24 , n. 3 , pp. 343-361.

El profesional de la información (EPI)

http://www.elprofesionaldelainformacion.com

Ellison, Nicole; Steinfield, Charles; Lampe, Cliff. "The benefits of Facebook 'friends': social capital and college students' use of online social network sites". J. comput.-mediated commun., 2007, v. 12, n. 4, pp. 1143-1168.

Fortunati, Leopoldina. "The mobile phone: new social categories and relations". En: Sosiale konsekvenser av mobiltelefoni, Oslo, 16 June 2000.

Foth, Marcus; Hearn, Greg. "Networked individualism of urban residents. Discovering the communicative ecology in inner-city apartment buildings". Information, communication \& society, 2007, Oct., v. 10, n. 5, pp. 749-772.

DOI: $10.1080 / 13691180701658095$

Habermas, Jürgen. The structural transformation of the public sphere. Cambridge, UK: Polity, 1989.

Henderson, Scott. "The social network, support and neurosis: the function of attachment in adult life". British $j$. psychiatry, 1977, n. 131, pp. 185-191.

Hill, Russell A.; Dunbar, Robin I. M. "Social network size in humans". Human nature, v. 14, n. 1, pp. 53-72.

http://www.liv.ac.uk/evolpsyc/Hill_Dunbar_networks.pdf

International Registry of Authors - Links to Identify Scientists (IraLIS)

http://www.iralis.org

Kraut, Robert E.; Patterson, Michael; Lundmark, Vicki; Kiesler, Sara; Mukhopadhyay, Tridas; Scherlis, William. "Internet paradox: A social technology that reduces social involvement and psychological well-being?" American psychologist, 1998, v. 53, n. 9, pp. 1017-1032.

Kraut, Robert E.; Kiesler, Sara; Boneva, Bonka; Cummings, Jonathon; Hegelson, Vicki; Crawford, Anne. "Internet para- dox revisited". Journal of social issues, 2002, v. 58, n. 1, pp. 49-74.

http://www.webuse.umd.edu/webshop/resources/kraut. $p d f$

Lin, Chieh-Peng; Bhattacherjee, Anol. "Understanding online social support and its antecedents: A socio-cognitive model". The social science journal, 2009, n. 46, pp. 724-737. doi 10.1016/j.soscij.2009.03.004

Lorente, Santiago. "Youth and mobile telephones: more than a fashion". En: Juventud y teléfonos móviles, S. Lorente (Ed.), Madrid: Ministerio de Trabajo y Asuntos Sociales, Injuve, 2002.

Orduña-Malea, Enrique; Rodríguez-Gairín, Josep-Manuel; Baiget, Tomàs. "Directorio de expertos en el tratamiento de la información (EXIT). Análisis de uso". El profesional de la información, 2007, sep.-oct., v. 16, n. 5, pp. 497-509.

DOI: 10.3145/epi.2007.sep.13.

Peset, Fernanda; Ferrer-Sapena, Antonia; Baiget, Tomàs. "Evolución social y networking en la comunidad biblio-documental". El profesional de la información, 2008, nov.-dic., v. 17, n. 6, pp. 627-635.

DOI: 10.3145/epi.2008.nov.05

Porak, Victor; Geissler, Ulrike; Einwiller, Sabine. "Corporate media - an approach for corporate community management". En: Proc of the 8th European conf on information systems, 2000, 8 pp.

http://is2.Ise.ac.uk/asp/aspecis/20000080.pdf

Punset, Eduard. Algo está cambiando en el modo de relacionarnos.

http://www.eduardpunset.es/5386/general/algo-esta-cam biando-en-el-modo-de-relacionarnos

Rice, Ronald E.; Shepherd, Adrian; Dutton, William H. "Social interaction and the internet. A comparative analysis of surveys in the US and Britain" (Chapter 2). En: The Oxford handbook of internet psychology, Joinson, Adam, et al. (eds.), Oxford University Press, 2007.

Rogers, Paul; Lea, Martin. "Social presence in distributed group environments: the role of social identity". Behaviour \& information technology, 2005, Mar.-Apr., v. 24, n. 2, pp. 151-158.

DOI: $10.1080 / 01449290410001723472$

Spears, Russell; Lea, Martin. "Social influence and the influence of the 'social' in computer-mediated communication". In: Martin Lea (ed.) Contexts of computer-mediated communication. Hemel Hempsted: Wheatsheaf, pp. 30-65.

Srivastava, Lara. "Mobile phones and the evolution of social behaviour". Behaviour \& information technology, 2005, Mar.-Apr., v. 24, n. 2, pp. 111-129.

Valkenburg, Patti M.; Peter, Jochen; Schouten, Alexander P. "Friend networking sites and their relationship to adolescents' well being and social self-esteem". Cyberpsychology and behavior, 2006, v. 9, pp. 584-590.

Walther, Joseph B.; Boyd, Shawn. Attraction to computermediated social support.

https://www.msu.edu/ jwalther/docs/support.html 